\title{
Anxiety and depression in patients with end-stage renal disease: impact and management challenges - a narrative review
}

This article was published in the following Dove Press journal: International Journal of Nephrology and Renovascular Disease

\section{Zhong Sheng Goh \\ Konstadina Griva}

Lee Kong Chian School of Medicine, Nanyang Technological University, Singapore
Correspondence: Konstadina Griva Clinical Sciences Building, Lee Kong Chian School of Medicine, Nanyang Technological University, II Mandalay Road, Singapore 308232

Tel +6569047348

Email konstadina.griva@ntu.edu.sg

\begin{abstract}
Psychiatric disorders commonly co-exist with the diagnosis of chronic kidney disease (CKD). Research on depression and CKD has increased to a great extent. Multiple studies have demonstrated that depression is more prevalent in CKD and that end-stage renal disease is a robust risk factor for adverse outcomes such as hospitalization and mortality, yet these are often underdiagnosed or untreated. This review provides a selective overview on the prevalence rates of depression and anxiety in patients with CKD and across renal replacement therapies, the factors most consistently associated with symptoms of distress and their clinical implications. Finally, treatment and management strategies from relevant literature are appraised and discussed. Keywords: psychological, difficulties, symptoms, chronic kidney disease
\end{abstract}

\section{Background}

Chronic kidney disease (CKD) affects $\sim 10-15 \%$ of the adults globally. ${ }^{1}$ The prevalence of this debilitating disease is projected to increase further due to the worldwide diabetes epidemic. ${ }^{2}$ In stages $1-4$ of CKD, treatment (ie, pharmacotherapy and diet) is focused on slowing the progression of kidney disease and preventing or treating complications and comorbid conditions. In the final stage of CKD (ie, stage 5 or endstage renal disease [ESRD]), renal replacement therapy (RRT) such as dialysis or kidney transplantation becomes necessary to maintain life. With increasing number of people developing CKD, patients requiring RRT is set to increase exponentially. This can cause increased burden on health care system.

All chronic diseases are often challenging to adjust to. Patients with CKD are required to make ongoing psychological adjustments over the course of their disease, such as accepting the life-threatening diagnosis and need for lifelong treatment, learning dialysis techniques, integrating treatment into their lives, and coping with treatment transitions/failures, side effects, and complications. ${ }^{3}$ In light of such a substantial and sustained disease burden, the management of CKD/ESRD has expanded from strictly clinical end points toward maintenance of quality of life (QoL), from diagnosis through to end of life care. Of paramount importance are psychological concerns related to the disease and associated renal treatment.

A plethora of studies have demonstrated jarring links between depression and anxiety and CKD/ESRD. Concurrently, psychological comorbidities have been documented to also affect treatment. ${ }^{4-6}$ It is thus important to understand the interaction of such psychological comorbidities and CKD outcomes. In this review article, we provide a selective narrative overview regarding the recent studies on rates of depression and 
anxiety in various renal treatment modalities, key associative factors, and potential interventions. It is not intended as a systematic literature but as a scoping synthesis of both empirical evidence and prior systematic reviews to highlight what is already known and directions of practice research. Relevant studies and reviews exploring depression and anxiety in patients with CKD were identified by searching the following databases: PubMed, PsycINFO, and Scopus. Search terms that were used included variations of the terms: depression; anxiety; kidney disease; ESRD; and dialysis. A selective set of studies are included to highlight evidence on the spectrum of CKD including early stage CKD and ESRD treated with different RRTs.

\section{Prevalence rates of depression and anxiety}

Some key questions regarding mood disorders (ie, depression and anxiety) in CKD relate to its prevalence relative to the other conditions and the general population, to various renal treatment modalities, and also relate to the course of CKD. There is wide variability among studies related to aforementioned topics and are mostly attributed to methodological differences in measurement and criteria to define depression and anxiety disorders (eg, self-reported vs diagnostic interviews), which will be discussed next.

Depression is an emotional state characterized by somatic and cognitive symptoms including feelings of sadness, worthlessness, sleeplessness, loss of appetite and sexual desires, and interest in usual activities. ${ }^{7}$ A clinical diagnosis of depression, most often major depressive disorder, is performed when symptoms of depression become persistent, often for more than 2 weeks. The assessment of depression is rather challenging in the CKD population, partly because of overlapping physical symptoms of uremia and depression, such as fatigue, loss of appetite, sleep disruption, and so on. Furthermore, other medical comorbidities such as sleep apnea and vascular complications, which are common in $\mathrm{CKD} / \mathrm{ESRD}$, might contribute to these symptoms as well.

Depression can be assessed through either structured clinical interviews, or self- or clinician-administered validated rating scales. Structured clinical interviews are generally seen as the "gold-standard" in the diagnosis of depressive disorders as they are based on specific diagnostic criteria (eg, Structured Clinical Interview for Diagnostic and Statistical Manual of Mental Disorders [SCID]). However, depressive symptoms are usually assessed by self- or clinician-administered validated rating scales such as the Beck's Depression Inventory (BDI) scale, Patient Health Questionnaire (PHQ), and Hospital Anxiety Depression Scale (HADS). These assessments are generally preferred in clinical settings for screening and in clinical research for pragmatic reasons to reduce time requirements and financial costs. ${ }^{8}$ While these screening tools are not diagnostic measures, they are helpful in identifying those who have significant symptoms and may require further evaluation. There are general cutoffs to signify threshold for caseness (as validated in clinical interviews) and proposed renal specific cutoffs for some screening instruments (ie, HADS, BDI).

Some studies have demonstrated higher prevalence rates of depression in patients with CKD/ESRD than that of other chronic diseases. ${ }^{9,10}$ For instance, in a study by Kimmel et al, the rates of hospitalization due to psychiatric illnesses among patients with ESRD aged 65 years and older were significantly higher for those with ESRD than those with ischemic heart disease, cerebrovascular disease, and peptic ulcer disease. ${ }^{11}$ As Cukor et al describes, there are unique, psychosocial paradigms of ESRD challenges that patients face which could explain the high prevalence of depression and anxiety as compared to other illnesses. ${ }^{3}$ When compared with the general population, patients with ESRD show more than five times the rate of suffering from depression. ${ }^{12}$ The reported prevalence rate of depression in patients with CKD ranges from $20 \%$ to $30 \% .^{13,14}$ Notably, the assessment method used to identify depression can affect the prevalence estimates. This was illustrated in a meta-analysis of 249 studies conducted by Palmer et al. ${ }^{4}$ Within patients undergoing dialysis, the prevalence rates of depression were found to be $22.8 \%$ using clinical interviews (eg, SCID and Diagnostic Interviews). However, when self- or clinician-rated questionnaires were used (eg, BDI and HADS), the prevalence rates were found to be $39.3 \%$, which was statistically higher than the previous figure.

Anxiety is another commonly co-occurring psychopathology with CKD/ESRD. Anxiety is an emotional state in which the individual experiences intense fear, uncertainty, and dread from the anticipation of a threatening situation. Anxiety disorders, unlike brief anxiety states caused by stressful events, last at least 6 months, are pervasive and can get worse without treatment. Compared to depression, however, the links between anxiety and CKD are relatively less studied. While anxiety disorders can be reliably diagnosed with the SCID or the Mini International Neuropsychiatric Interview (MINI), they are often assessed with much briefer, but well-validated, scales such as the HADS, Beck's Anxiety Inventory (BAI) scale, and Generalized Anxiety Disorder 7 Inventory (GAD-7). 
Existing literature points to increased levels of anxiety in patients with CKD. The prevalence rate of anxiety in patients with ESRD was estimated to be around $12 \%$ to $52 \%$ in a previous study. ${ }^{5}$ However, the exact prevalence remains unclear, mostly due to the limited number of studies, different sample population, and different screening methods used. Studies that have used SCID to diagnose anxiety disorders have found prevalence rates ranging from $0 \%$ to $45.7 \% .{ }^{14-16}$

As illustrated, the prevalence rates of depression and anxiety in patients with CKD/ESRD may not be exactly well defined. However, the high levels of anxiety and depression in these patients remain alarming.

\section{The course of depression and anxiety}

Studies have found evidence of persistent symptoms of depression and anxiety over the course of CKD. Longitudinal studies, albeit few, have shown that emotional symptoms are likely to fluctuate considerably over the course of CKD because of life circumstances, illness burden, disease stages, treatment factors, and other psychosocial issues.

A recent, longitudinal study by $\mathrm{Ng}$ et al followed up with 159 patients undergoing dialysis over a period of 12 months. ${ }^{17}$ According to their results, 63 patients (39.6\%) presented with persistent symptoms of depression, whereas 50 patients $(31.8 \%)$ had persisting anxiety. Around $13 \%$ of the patients reported diminished symptoms, but another $13 \%-18 \%$ had new onset of symptoms. Diminishing perceived social support was found to be associated with increased and persisting distress over time. Similar patterns were also observed in other studies. ${ }^{14,18}$ Prospective work hence highlight that depression and anxiety for the majority of patients with CKD is chronic, with symptoms persisting over time and course of disease.

\section{Depression and anxiety and RRT modalities}

Few studies have evaluated the prevalence of depression and outcomes in patients with early stage CKD (ie, stages 1-4), patients established on peritoneal dialysis (PD), or patients with kidney transplant. Research on symptoms of anxiety in these patient subgroups is even more scarce.

Evidence indicates that depression rates in patients with early stage CKD not requiring RRT, albeit lower, are not negligible. Several studies have established an association between depression and CKD based on impaired estimated glomerular filtration rate (eGFR). Hedayati et al found a $21 \%$ point prevalence of major depression among veterans with eGFR $<90 \mathrm{~mL} / \mathrm{min} / 1.73 \mathrm{~m}^{2} .{ }^{19}$ Similarly, the prevalence of at least moderate depressive symptoms was $26 \%$ among subjects with hypertensive CKD in the African-American Study of Kidney Disease and Hypertension Cohort Study. ${ }^{20}$ The Cardiovascular Health Study found that in their community-based cohort of elderly subjects, those with depressive symptoms had 19\% greater odds of impaired eGFR (95\% CI=1.01-1.40) compared to those without, after adjustment for demographics, clinical variables, and health behaviors. ${ }^{21}$

\section{Depression in patients with ESRD undergoing PD}

PD is a home-based dialysis modality that involves the insertion of dialysis fluid into the patient's peritoneal cavity for the filtration of toxins and water through the peritoneum. The PD process requires patient to do the dialysate exchange either three times a day (for continuous ambulatory peritoneal dialysis [CAPD]) or while sleeping (for automated peritoneal dialysis [APD]). Although PD affords greater opportunities for control, autonomy, and the convenience of home-based treatment, recent studies have reported disconcertingly high levels of depression in patients maintained on PD regime. ${ }^{22}$ The estimated prevalence rates range from $18.7 \%$ to $51.5 \%$, depending on the measurement and the criteria used for diagnosis. ${ }^{23-25}$

There also seem to be differences across the PD modalities. Griva et al noted that patients performing the PD procedure manually (CAPD) perceived more treatment associated disruption and higher rates of depression relative to patients on the automated APD. ${ }^{24}$ This could be due to the more regular daytime exchange of dialysate for patients on the manual CAPD, and thus less time for work, family, and social life as compared to patients on night-time APD. ${ }^{26}$

Whether PD confers better psychological outcomes than HD is still largely contentious. In some studies, it has been reported that patients with HD experience higher symptoms of depression and anxiety than that of patients on $\mathrm{PD},{ }^{27,28}$ in line with dialysis modality differences in QoL. ${ }^{29}$ Patients with HD have also been shown to have higher hospitalization rates for depression and affective disorders. ${ }^{11}$ Distress in HD may be related to procedural aspects of treatment such as the need to travel to dialysis centers (DCs), transportation issues, spending considerable time in medical environments (ie, dialysis wards), and the prolonged sitting time during dialysis. Whereas the dialysis units may foster social support among patients, issues related to emotional contagion among them or witnessing adverse outcomes in others may trigger distress. Distress may also be related to fear of access 
complications, dialysis-related symptoms, and side effects, which are common concerns among patients on HD. ${ }^{30}$

In contrast, other studies have noted that depression and anxiety in patients on PD are equal or even higher than those reported for patients on HD. ${ }^{25,31}$ By virtue of PD being a home-based dialysis technique, individuals may feel sustained distress over the daily responsibility of their own health and well-being or medicalization of home environment that may act as constant reminder of their ill health. ${ }^{32}$ Compared to patients on HD, patients on PD lack regular contact with hospital staff who can provide reassurance of quality care for each session, and a point of reference for their queries and concerns. Opportunities for patient peer support are limited compared to those afforded for patients on HD in the DCs. Distress may also be triggered by the worry about burden to family due to home-based PD. It is to be noted though that symptoms of depression and anxiety are comparable for patients on self-care PD (i.e. patients administering dialysis unaided) and those on assisted PD (PD administration involving paid or unpaid caregivers), so as reports of burden among family members and caregivers. ${ }^{24,33}$ The continuous nature of PD regimen and increased time requirements compared to intermittent HD may adversely affect emotional adjustment and, over time, may take its toll and manifest as depression and burn out. . $^{2434}$

\section{Depression and transplantation}

Kidney transplantation is the preferred RRT as it affords superior clinical outcomes and QoL but is constrained by the limited availability of grafts. In most settings, there is a long waiting list of kidney transplant candidates. ${ }^{35}$

The process of kidney transplantation can be stressful for patients both before and after receipt of kidney. ${ }^{36}$ While at the waitlist, patients undergo medical and psychosocial evaluation to ensure suitability. A systematic review investigating waitlist patients' experiences found that although being put on the waitlist offered hope, it also introduced negative feelings such as anxiety, disillusionment, disappointment, and vulnerability when waiting time was prolonged. ${ }^{37}$ After transplant, patients face new challenges such as the need to adhere to immunosuppressive medications, diet restrictions, and the inherent fear of graft rejection, or death.

In a recent review conducted by Veater and East, kidney transplant patients were noted to experience elevated levels of depression as compared to the general population but generally lower rates of depression compared to patients on other RRTs. ${ }^{6}$ Thus, despite the ability of kidney transplants to restore an individual's level of functioning, there is evidence of persistent symptoms of psychological distress.

\section{Consequences of depression and anxiety on treatment outcomes}

In general, the presence of psychiatric disorders has been shown to be associated with a higher likelihood of adverse clinical outcomes in patients with CKD. Longitudinal studies have demonstrated that for patients on HD, those who had a clinician diagnosis of depression have worse clinical endpoints such as higher rates of hospitalization and inpatient days, higher rates of dialysis withdrawal and mortality, ${ }^{19,38,39}$ and increased use of health care system. ${ }^{40}$

Mapes et al demonstrated that among patients on HD, depression was independently associated with increased mortality and hospitalization. ${ }^{41}$ Several other original studies and systematic reviews have confirmed the prognostic association of depression with overall mortality. A recent meta-analysis by Farrokhi et al which included 31 studies and data of more than 67,000 patients undergoing dialysis found that the presence of depressive symptoms in such patients is associated with a $50 \%$ increase in the risk of mortality. ${ }^{42}$ Notably, the method of measuring depression (self-rating scale vs interview or physician assessment) did not appear to modify the association between depression and mortality. ${ }^{43}$

Of greater clinical relevance are persistent symptoms of depression. One study in which depression was measured at 6-monthly intervals in 917 incident patients undergoing dialysis for 2 years found that persistent or recurrent depression was associated with cardiovascular mortality, whereas depression detected over 6 months previously or at baseline only was not associated with mortality. This indicates that persistent depression may be associated with death but resolved depression may no longer have ongoing prognostic implications. ${ }^{44}$

In patients with CKD not requiring dialysis, depression has been shown to be associated with the rate of progression to ESRD. One prospective observational study concluded that depressive symptoms in CKD predicted increased rate of progression to ESRD, and faster dialysis initiation, death, or hospitalization. ${ }^{45}$ The results of this study echoed another landmark study by Hedayati et al which found patients with both a CKD and a clinical diagnosis of depression twice more likely to be hospitalized and more than three times likely to progress to ESRD and dialysis than patients who are nondepressed. ${ }^{46}$ This association was found to be independent 
of disease severity and the presence of other comorbidities such as diabetes.

Depressive symptoms have also been shown to affect kidney transplant outcomes. Patients who had symptoms of depression after kidney transplant were more likely to die than patients without depression. A prospective cohort study of 840 transplanted patients over 5-year period found a higher mortality rate in patients with depression as compared to patients without $(21 \%$ vs $13 \% ; p=0.004) .{ }^{47}$ Psychiatric symptoms could also affect the likelihood of patients who are put on the kidney transplant waiting list. Bayat et al found that the presence of a psychiatric disorder was a strong, independent factor in determining access to the transplant waitlist. ${ }^{48}$ Similarly, Szeifert et al found that patients who reported lower levels of depression on the CES-D had a higher likelihood of being placed on the renal transplant waitlist. However, this association was not found for patients already on the waitlist who are waiting to receive a kidney transplant. ${ }^{49}$

In patients undergoing $\mathrm{PD}$, depression and anxiety symptoms have been noted to increase the likelihood of adverse outcomes. As compared to their nondepressed counterparts, patients on PD who are depressed are associated with higher risk of inflammation and cardiovascular disease and higher mortality rates. ${ }^{50}$ Depressed patients on PD are also at a higher risk of developing peritonitis, or the infection of the peritoneum, which in severe cases can result in death. Juergensen et al, for instance, have demonstrated that patients on PD who reported higher scores for anxiety and depression were more likely to develop peritonitis within the following 12 months. ${ }^{51}$ Likewise, Troidle et al in their prospective study of 162 patients on $\mathrm{PD}$ found that patients who had BDI $\geq 11$ at baseline had 2.7 times more likelihood of developing peritonitis as compared to patients with no depression. ${ }^{23}$

Clinical outcomes associated with anxiety have been far less studied than those of depression. The presence of anxiety disorder in patients on HD has been observed to result in a significantly lower QoL than patients with no psychopathology, and an even lower QoL than patients with only a depressive disorder. ${ }^{14,52}$ In terms of clinical outcomes, few small studies have found a trend of anxiety symptoms leading to adverse clinical events. The presence of anxiety symptoms could have an influence on patients with CKD who did not require dialysis at first. For instance, Loosman et al found that among patients with CKD who are not yet on dialysis, the presence of anxiety symptoms shows a trend toward death or dialysis initiation. ${ }^{53}$

\section{Mechanism linking depression to outcomes}

One of the key concerns regarding the association of depression with outcomes is whether depression itself plays a direct role in adverse clinical outcomes or that the depressive symptoms are merely indicators of increased comorbidity and disease severity. Although not well characterized, two mechanisms by which depression is associated with adverse renal outcomes have also been proposed.

First, the link between depression and adverse outcomes may be due to biological factors. Genetic influences of depression and ischemic heart disease in twin studies and a lower heart rate variability were observed in patients with depression, both of which lead to higher likelihood of mortality. Allostatic dysregulation from depression and CKD progression has also been observed to cause hyperactivity of the hypothalamic-pituitary axis activity, resulting in increased cortisol and norepinephrine excretion. ${ }^{3,54}$ This can lead to alterations in malnutrition, immunity, inflammation, and atherosclerosis states. ${ }^{55}$ This theory was also supported by early evidence that depression could be involved in the development of malnutrition, inflammation, and atherosclerosis (MIA) in patients undergoing dialysis. ${ }^{56}$

Second, depression might also be associated with clinical outcomes via behavioral pathways. Depression has been associated with decreased adherence to dialysis, prescribed medications, and dietary restrictions in patients on chronic $\mathrm{HD}$, which in turn are predictive of poor medical outcomes and higher mortality. ${ }^{57-59}$ In addition to nonadherence, other poor health behaviors, such as physical inactivity, smoking, dietary indiscretions, which tend to cluster together in individuals with depression in general may also be implicated. At this point, it is also useful to note that the risks of selfharm and suicide are elevated in patients with dialysis who have depression. ${ }^{60}$

\section{Factors associated with depression and anxiety}

Multiple studies have examined possible social, clinical, and psychological factors associated with depression in patients with CKD/ESRD. For instance, a recent literature review by Veater and East investigating depression in patients undergoing renal transplantation identified several risk factors for developing depression including gender, sleep quality, employment, and financial status. ${ }^{6}$

Several sociodemographic factors have been identified to cause anxiety and depression. Females were observed to have 
a tendency of developing anxiety, whereas males develop depression. ${ }^{61}$ In terms of ethnicity, white patients, as compared to African-American patients, were at higher risk of depression. ${ }^{11,62}$ In a study by Yoong et al in Singapore, Chinese patients were found to be more likely to develop depression than those of Malay and other ethnicities ${ }^{63}$ Evidently, ethnic culture could have an influence on the propensity for psychiatric difficulties in CKD.

Patients who had lower levels of education and those who are unemployed were also observed to be at higher risks. Presumably due to do an overall lower socioeconomic profile, problems in psychological well-being, social relationships, and general health are more prevalent in these subgroups of patients. ${ }^{64,65}$ Finally, those who were divorced or widowed reported higher levels of depression. ${ }^{66}$ This is possibly due to the perceived lack of social support and lower QoL as a result of their divorce or widowing.

The risk of depression and anxiety in patients with CKD has also been associated with various clinical parameters. Patients with a history of psychiatric illness were found to be more prone to develop depression after renal transplantation. ${ }^{19,67,68}$ Comorbidity might also increase the risk of development of depression and anxiety. For instance, patients with depressive symptoms were also found to have other comorbid illnesses such as diabetes mellitus, hypertension, cardiovascular heart disease, hypoalbuminemia, and substance abuse. ${ }^{8,61,62}$

An increased risk of depression and anxiety has also been associated with adverse outcomes in kidney transplantation. Kidney graft rejection has been reported to trigger feelings of depression, anxiety, aggression, and loss. ${ }^{69}$ In a qualitative study of patients who experienced kidney graft failure, the patients reported periods of grief and loss for the "imagined future." These psychological feelings of loss were unfortunately often under-recognized by health care professionals. ${ }^{70}$

There is mounting evidence of an association between illness perceptions and depression. ${ }^{18,71}$ Illness perceptions are conceptualized within a framework of self-regulation (Common-Sense Model [CSM]). ${ }^{72}$ CSM posits that a person's illness beliefs and understanding of the health threat (ie, their cognitive illness representation) and parallel emotional responses to illness inform coping behaviors and in turn influence outcomes such as physical and emotional health. Major components include perceptions of how the illness was caused, symptoms experienced, the duration (timeline), the consequences of the illness are for life, the symptoms associated with the illness (identity), and how the condition is controlled or cured by one's own behavior or treatment.
The CSM has been extended with "common-sense" beliefs about treatment because treatment is an important facet of the illness trajectory. This is particularly true for CKD/ESRD where dialysis has a major impact on patients' daily life.

The relationship between depression and illness/treatment cognitions has been studied most frequently, whereas studies in relation to anxiety or other mood disorders are scarce. Overall, cognitive illness representations were associated with poor emotional health in the theoretically expected direction. Associations were found for both patients with CKD not requiring RRT and patients with ESRD already on RRT.

In a recent review of studies in CKD patients not requiring dialysis, Clarke et $\mathrm{al}^{73}$ noted that symptoms of depression and anxiety are more correlated with illness perception than with health status, which supports the CSM.

For patients already on RRT, depression was found to be associated with illness perception in terms of perceived locus of control and illness intrusiveness. In a study by Christensen et al, a strong internal locus of control was associated with less depression in patients who did not experience a failed renal transplant. ${ }^{74}$ This association was, however, inversed for patients who previously had a failed renal transplant (ie, high internal locus of control led to greater symptoms of depression). In terms of illness intrusiveness, patients who perceived greater disruption to their lives as a result of the renal disease experienced increased depression and distress. ${ }^{75,76}$

\section{Management and treatment}

Given the high prevalence and dire consequences of depression, the nephrology community has long recognized that treatment goals should focus on early identification and remission or improvements of distress symptoms, including depression, anxiety, and general emotional well-being. According to the K/DOQI guidelines, the psychological status of each patient undergoing dialysis should be evaluated by a social worker upon initiation of dialysis and then at least biannually, with specific focus on the presence of depression and anxiety. ${ }^{77}$ Qualitative studies have revealed that patients consider the psychosocial aspects and reduction in symptoms of CKD as priorities for social scientific research agendas, ${ }^{78,79}$ thus suggesting that the optimal management of psychiatric symptoms in ESRD is important and necessary.

Yet, there is little evidence available that can guide clinicians about the most effective treatment options for the several mood conditions. Data suggest that depression is highly undertreated and underdiagnosed in patients with ESRD. A Dialysis Outcomes and Practice Patterns Study 
(DOPPS) by Lopes et al found that antidepressants were prescribed to only $34.9 \%$ patients on $\mathrm{HD}$ who had a physician diagnosis of depression. ${ }^{80}$

Psychopharmacological interventions are commonly cited as a method to alleviate depressive symptoms of patients with ESRD. However, clinical trials so far have employed small sample sizes, and evidence on benefits and harms of antidepressant therapy in ESRD remains inconclusive. Antidepressant medications, most commonly those in the class of selective serotonin reuptake inhibitors (SSRIs), ${ }^{81}$ have been demonstrated to improve symptoms. However, there are concerns as to whether their side effects can be well tolerated..$^{82}$ As medication count is high among patients with $\mathrm{CKD} / \mathrm{ESRD}$, adherence to pharmacological treatments may be more problematic in this population.

Therefore, pragmatic and evidence-based psychological interventions require evaluation. In this regard targeting unhelpful illness and treatment perceptions and enhancing opportunities for social networking and support might be an effective approach in order to improve symptoms of depression. Recent work indicates that cognitive behavioral therapy (CBT) might be an efficacious, acceptable, and practical treatment option for depressive symptoms in patients undergoing dialysis. ${ }^{83,84}$ Cognitive behavior therapy aims to counteract psychological disorders or problems that arise from dysfunctional thoughts, feelings, and behaviors. ${ }^{85}$ In the context of depression, negative perceptions of the "self, experience, and future" are maintained through selective information processing that focuses attention on, and thus recall of, information that reinforces it. Patients are taught to modify thoughts that are not consistent with evidence, change maladaptive behaviors, and develop skills for coping with negative feelings.

However, such programs may be hard to integrate into standard clinical care. To this end, it may be worthwhile to evaluate more feasible ways of providing support, such as nurse or peer-support programs. Clinic integrated, or telephone delivered, CBT that have been shown to be effective in other patient populations can be realistically implemented in clinical practice. Recent developments in telehealth or e-health have seen the integration of psychotherapeutic interventions for distressed patients going online. A recent framework developed by Hudson et al established the feasibility of online CBT treatments for managing comorbid distress and ESRD. ${ }^{86}$ The self-help protocol that aims to improve distress worked based on the CSM, which targets patient's illness beliefs and self-efficacy. While future work has to be conducted to establish its efficacy, the proposed framework was found to be feasible and effective.

Other practical approaches such as stress management training (eg, mindfulness meditation) and enhancing communication with staff and social support of patients and caregivers may also be important components of mental health services supporting patients with CKD. Training of renal health care providers, especially nurses who have the most frequent contact with some patient groups in mental health issues and brief interventions may be particularly useful as the first step toward integrated care models and stepped-care approaches.

Overall, there is a clear need to provide a holistic care for patients with ESRD both physically and psychologically. The current National Institute for Health and Care Excellence (NICE) guidelines for depression in adults with a chronic physical health problem, such as CKD/ESRD, recommend collaborative care in a "stepped-care framework" in which to organize health services. ${ }^{87}$ Patients with an inadequate response to one or more treatments are "stepped up" from low-intensity care to a more intensive form of management (including lifestyle, psychological, and pharmacological therapies). As Finkelstein et al describes, systematic strategies that involve screening, diagnosing, and treating of patients with CKD and caregivers for depression are important to manage the psychological impacts that this disease have on their lives. ${ }^{88}$

\section{Summary}

Patients with CKD often have psychiatric difficulties in the form of depression and anxiety. Existing literature sheds light onto the specific impacts and mechanisms that psychiatric difficulties can influence CKD treatment and outcomes. If overlooked, these psychiatric comorbidities can affect the patient's treatment prognosis and mortality. There is an everincreasing need for physicians to address and treat patients with CKD that present with psychiatric comorbidities.

The high prevalence rates of depression and anxiety in patients with CKD as compared to other chronic diseases is of an important concern. It is unfortunate that compared to other chronic illnesses such as coronary heart diseases and cancers, the study of CKD and related psychological disorders are far less extensive. Existing epidemiological studies have revealed important insights and factors that contribute to the development of depression and anxiety in patients with CKD. Patients with certain social, psychological, and clinical characteristics were found to be more prone to falling into 
depression. These associations provide practitioners vital indicators to identify and assess patients for psychiatric disorders more efficiently.

Implementing systematic distress screening in routine clinical care would be an important first step, which should be performed in conjunction with providing appropriate interventions. Treatment modalities for comorbid depression in patients with CKD have shown promising, albeit preliminary, results. Pharmacotherapies and psychotherapies are among the few interventions that can help relieve patients of their symptoms. Pharmacological treatment for patients with depression in the presence of CKD needs additional attention to avoid medical interactions with the already large number of renal medications. Extra care must be given to these patients to promote treatment adherence and address unhelpful illness and treatment perceptions.

It would also be important to more broadly consider the spectrum of emotional experience including anxiety, worrying, fear of progression of kidney disease, and fear of the future in general, demoralization, death and dying, hopelessness, questions around the meaning of life, and the experience of recurrent psychological and physical trauma through the disease trajectory, just to name a few.

\section{Disclosure}

The authors report no conflicts of interest in this work.

\section{References}

1. Coresh J, Selvin E, Stevens LA, et al. Prevalence of chronic kidney disease in the United States. JAMA. 2007;298(17):2038-2047.

2. Tabish SA. Is diabetes becoming the biggest epidemic of the twenty-first century? Int J Health Sci (Qassim). 2007;1(2):V-VIII.

3. Cukor D, Cohen SD, Peterson RA, Kimmel PL. Psychosocial aspects of chronic disease: ESRD as a paradigmatic illness. J Am Soc Nephrol. 2007;18(12):3042-3055.

4. Palmer S, Vecchio M, Craig JC, et al. Prevalence of depression in chronic kidney disease: systematic review and meta-analysis of observational studies. Kidney Int. 2013;84(1):179-191.

5. Murtagh FE, Addington-Hall J, Higginson IJ. The prevalence of symptoms in end-stage renal disease: a systematic review. Adv Chronic Kidney Dis. 2007;14(1):82-99.

6. Veater NL, East L. Exploring depression amongst kidney transplant recipients: a literature review. J Ren Care. 2016;42(3):172-184.

7. Davison GC, Neale JM. Abnormal Psychology. 8th ed. New York: John Wiley; 2004.

8. Hedayati SS, Bosworth HB, Kuchibhatla M, Kimmel PL, Szczech LA. The predictive value of self-report scales compared with physician diagnosis of depression in hemodialysis patients. Kidney Int. 2006;69(9):1662-1668.

9. Baumeister H, Härter M. Mental disorders in patients with obesity in comparison with healthy probands. Int J Obes (Lond). 2007;31(7): 1155-1164.

10. Ormel J, von Korff M, Burger H, et al. Mental disorders among persons with heart disease - results from World Mental Health surveys. Gen Hosp Psychiatry. 2007;29(4):325-334.
11. Kimmel PL, Thamer M, Richard CM, Ray NF. Psychiatric illness in patients with end-stage renal disease. Am J Med. 1998;105(3): 214-221.

12. Hedayati SS, Jiang W, O'Connor CM, et al. The association between depression and chronic kidney disease and mortality among patients hospitalized with congestive heart failure. Am J Kidney Dis. 2004;44(2):207-215.

13. Hedayati SS, Finkelstein FO. Epidemiology, diagnosis, and management of depression in patients with CKD. Am J Kidney Dis. 2009;54(4):741-752.

14. Cukor D, Coplan J, Brown C, et al. Depression and anxiety in urban hemodialysis patients. Clin J Am Soc Nephrol. 2007;2(3):484-490.

15. Cukor D, Coplan J, Brown C, et al. Anxiety disorders in adults treated by hemodialysis: a single-center study. Am J Kidney Dis. 2008;52(1):128-136.

16. Preljevic VT, Østhus TB, Sandvik L, et al. Psychiatric disorders, body mass index and C-reactive protein in dialysis patients. Gen Hosp Psychiatry. 2011;33(5):454-461.

17. Ng HJ, Tan WJ, Mooppil N, Newman S, Griva K. Prevalence and patterns of depression and anxiety in hemodialysis patients: a 12-month prospective study on incident and prevalent populations. $\mathrm{Br} J$ Health Psychol. 2015;20(2):374-395.

18. Chilcot J, Norton S, Wellsted D, Davenport A, Firth J, Farrington K. Distinct depression symptom trajectories over the first year of dialysis: associations with illness perceptions. Ann Behav Med. 2013;45(1):78-88.

19. Hedayati SS, Minhajuddin AT, Toto RD, Morris DW, Rush AJ. Prevalence of major depressive episode in CKD. Am J Kidney Dis. 2009;54(3):424-432.

20. Fischer MJ, Kimmel PL, Greene T, et al; AASK study group. Sociodemographic factors contribute to the depressive affect among African Americans with chronic kidney disease. Kidney Int. 2010;77(11): 1010-1019.

21. Kop WJ, Seliger SL, Fink JC, et al. Longitudinal association of depressive symptoms with rapid kidney function decline and adverse clinical renal disease outcomes. Clin J Am Soc Nephrol. 2011;6(4):834-844.

22. Yu ZL, Yeoh LY, Seow YY, Luo XC, Griva K. Evaluation of adherence and depression among patients on peritoneal dialysis. Singapore Med J. 2012;53(7):474-480.

23. Troidle L, Watnick S, Wuerth DB, Gorban-Brennan N, Kliger AS, Finkelstein FO. Depression and its association with peritonitis in long-term peritoneal dialysis patients. Am J Kidney Dis. 2003;42(2):350-354.

24. Griva K, Davenport A, Harrison M, Newman S. An evaluation of illness, treatment perceptions, and depression in hospital- vs. home-based dialysis modalities. J Psychosom Res. 2010;69(4):363-370.

25. Griva K, Kang AW, Yu ZL, et al. Quality of life and emotional distress between patients on peritoneal dialysis versus community-based hemodialysis. Qual Life Res. 2014;23(1):57-66.

26. Bro S, Bjorner JB, Tofte-Jensen $\mathrm{P}$, et al. A prospective, randomized multicenter study comparing APD and CAPD treatment. Perit Dial Int. 1999;19(6):526-533.

27. Juergensen E, Wuerth D, Finkelstein SH, Juergensen PH, Bekui A, Finkelstein FO. Hemodialysis and peritoneal dialysis: patients' assessment of their satisfaction with therapy and the impact of the therapy on their lives. Clin J Am Soc Nephrol. 2006;1(6):1191-1196.

28. Theofilou P. Quality of life in patients undergoing hemodialysis or peritoneal dialysis treatment. J Clin Med Res. 2011;3(3):132-138.

29. Noshad H, Sadreddini S, Nezami N, Salekzamani Y, Ardalan MR. Comparison of outcome and quality of life: haemodialysis versus peritoneal dialysis patients. Singapore Med J. 2009;50(2):185-192.

30. Griva K, Li ZH, Lai AY, Choong MC, Foo MW. Perspectives of patients, families, and health care professionals on decision-making about dialysis modality - the good, the bad, and the misunderstandings! Perit Dial Int. 2013;33(3):280-289.

31. Lin YH, Yang Y, Chen SY, Chang CC, Chiu PF, Huang CY. The depression status of patients with end-stage renal disease in different renal replacement therapies. Int J Urol Nurs. 2011;5(1):14-20. 
32. Mittal SK, Ahern L, Flaster E, Mittal VS, Maesaka JK, Fishbane S. Self-assessed quality of life in peritoneal dialysis patients. Am JNephrol. 2001;21(3):215-220.

33. Griva K, Goh CS, Kang WC, et al. Quality of life and emotional distress in patients and burden in caregivers: a comparison between assisted peritoneal dialysis and self-care peritoneal dialysis. Qual Life Res. 2016;25(2):373-384.

34. Moore R, Teitelbaum I. Preventing burnout in peritoneal dialysis patients. Adv Perit Dial. 2009;25:92-95.

35. García-García G, Harden P, Chapman J; World Kidney Day Steering Committee 2012. The global role of kidney transplantation. Nefrologia. 2012;32(1):1-6. Spanish.

36. Terán-Escandón D, Ruiz-Ornelas J, Estrada-Castillo JG, Barajas-Juárez L, Díaz-Martínez A. Anxiety and depression among renal transplantation candidates: impact of donor availability. Actas Esp Psiquiatr. 2001;29(2):91-94 [Article in Spanish].

37. Tong A, Hanson CS, Chapman JR, et al. "Suspended in a paradox" patient attitudes to wait-listing for kidney transplantation: systematic review and thematic synthesis of qualitative studies. Transpl Int. 2015;28(7):771-787.

38. Hedayati SS, Bosworth HB, Briley LP, et al. Death or hospitalization of patients on chronic hemodialysis is associated with a physician-based diagnosis of depression. Kidney Int. 2008;74(7):930-936.

39. McDade-Montez EA, Christensen AJ, Cvengros JA, Lawton WJ. The role of depression symptoms in dialysis withdrawal. Heal Psychol. 2006;25(2):198-204.

40. Abbas Tavallaii S, Ebrahimnia M, Shamspour N, Assari S. Effect of depression on health care utilization in patients with end-stage renal disease treated with hemodialysis. Eur J Intern Med. 2009;20(4):411-414.

41. Mapes DL, Bragg-Gresham JL, Bommer JJ, et al. Health-related quality of life in the Dialysis Outcomes and Practice Patterns Study (DOPPS). Am J Kidney Dis. 2004;44(5 Suppl 2):54-60.

42. Farrokhi F, Abedi N, Beyene J, Kurdyak P, Jassal SV. Association between depression and mortality in patients receiving long-term dialysis: a systematic review and meta-analysis. Am J Kidney Dis. 2014;63(4): 623-635.

43. Palmer SC, Vecchio M, Craig JC, et al. Association between depression and death in people with CKD: a meta-analysis of cohort studies. Am J Kidney Dis. 2013;62(3):493-505.

44. Boulware LE, Liu Y, Fink NE, et al. Temporal relation among depression symptoms, cardiovascular disease events, and mortality in end-stage renal disease: contribution of reverse causality. Clin J Am Soc Nephrol. 2006;1(3):496-504.

45. Tsai YC, Chiu YW, Hung CC, et al. Association of symptoms of depression with progression of CKD. Am J Kidney Dis. 2012;60(1):54-61.

46. Hedayati SS, Minhajuddin AT, Afshar M, Toto RD, Trivedi MH, Rush AJ Association between major depressive episodes in patients with chronic kidney disease and initiation of dialysis, hospitalization, or death. JAMA 2010;303(19):1946-1953.

47. Novak M, Molnar MZ, Szeifert L, et al. Depressive symptoms and mortality in patients after kidney transplantation: a prospective prevalent cohort study. Psychosom Med. 2010;72(6):527-534.

48. Bayat S, Frimat L, Thilly N, Loos C, Briançon S, Kessler M. Medical and non-medical determinants of access to renal transplant waiting list in a French community-based network of care. Nephrol Dial Transplant. 2006;21(10):2900-2907.

49. Szeifert L, Bragg-Gresham JL, Thumma J, et al. Psychosocial variables are associated with being wait-listed, but not with receiving a kidney transplant in the dialysis outcomes and practice patterns study (DOPPS). Nephrol Dial Transplant. 2012;27(5):2107-2113.

50. Ko GJ, Kim MG, Yu YM, Jo SK, Cho WY, Kim HK. Association between depression symptoms with inflammation and cardiovascular risk factors in patients undergoing peritoneal dialysis. Nephron Clin Pract. 2010;116(1):c29-c35.

51. Juergensen PH, Juergensen DM, Wuerth DB, et al. Psychosocial factors and incidence of peritonitis. Adv Perit Dial. 1996;12:196-198.
52. Cukor D, Ver Halen N, Fruchter Y. Anxiety and quality of life in ESRD. Semin Dial. 2013;26(3):265-268.

53. Loosman WL, Rottier MA, Honig A, Siegert CE. Association of depressive and anxiety symptoms with adverse events in Dutch chronic kidney disease patients: a prospective cohort study. BMC Nephrol. 2015;16(1):155.

54. Honda H, Qureshi AR, Heimbürger O, et al. Serum albumin, C-reactive protein, interleukin 6 , and fetuin a as predictors of malnutrition, cardiovascular disease, and mortality in patients with ESRD. Am J Kidney Dis. 2006;47(1):139-148.

55. Taraz M, Taraz S, Dashti-Khavidaki S. Association between depression and inflammatory/anti-inflammatory cytokines in chronic kidney disease and end-stage renal disease patients: a review of literature. Hemodial Int. 2015;19(1):11-22.

56. Simic Ogrizovic S, Jovanovic D, Dopsaj V, et al. Could depression be a new branch of MIA syndrome? Clin Nephrol. 2009;71(2):164-172.

57. Kimmel PL, Peterson RA, Weihs KL, et al. Psychosocial factors, behavioral compliance and survival in urban hemodialysis patients. Kidney Int. 1998;54(1):245-254.

58. Kaveh K, Kimmel PL. Compliance in hemodialysis patients: multidimensional measures in search of a gold standard. Am J Kidney Dis. 2001;37(2):244-266.

59. Leggat JE Jr. Adherence with dialysis: a focus on mortality risk. Semin Dial. 2005;18(2):137-141.

60. Pompili M, Venturini P, Montebovi F, et al. Suicide risk in dialysis: review of current literature. Int J Psychiatry Med. 2013;46(1):85-108.

61. Hou Y, Li X, Yang L, et al. Factors associated with depression and anxiety in patients with end-stage renal disease receiving maintenance hemodialysis. Int Urol Nephrol. 2014;46(8):1645-1649.

62. Lopes AA, Bragg J, Young E, et al; Dialysis Outcomes and Practice Patterns Study (DOPPS). Depression as a predictor of mortality and hospitalization among hemodialysis patients in the United States and Europe. Kidney Int. 2002;62(1):199-207.

63. Yoong RK, Mooppil N, Khoo EY, et al. Prevalence and determinants of anxiety and depression in end stage renal disease (ESRD). a comparison between ESRD patients with and without coexisting diabetes mellitus. J Psychosom Res. 2017;94:68-72.

64. Sesso R, Rodrigues-Neto JF, Ferraz MB. Impact of socioeconomic status on the quality of life of ESRD patients. Am J Kidney Dis. 2003;41(1):186-195.

65. Vázquez I, Valderrábano F, Jofré R, et al; Spanish Cooperative Renal Patients Quality of Life Study Group. Psychosocial factors and quality of life in young hemodialysis patients with low comorbidity. J Nephrol. 2003;16(6):886-894.

66. Theofilou P. Depression and anxiety in patients with chronic renal failure: the effect of sociodemographic characteristics. Int J Nephrol. 2011;2011:514070.

67. Spencer BW, Chilcot J, Farrington K. Still sad after successful renal transplantation: are we failing to recognise depression? An audit of depression screening in renal graft recipients. Nephron Clin Pract. 2011;117(2):c106-c112.

68. Sensky T. Psychiatric morbidity in renal transplantation. Psychother Psychosom. 1989;52(1-3):41-46.

69. Ouellette A, Achille MA, Vachon M. Psychological impact of kidney graft failure and implications for the psychological evaluation of retransplant candidates. Dial Transplant. 2006;35(6):354-361.

70. Gill P, Lowes L. Renal transplant failure and disenfranchised grief: participants' experiences in the first year post-graft failure - a qualitative longitudinal study. Int J Nurs Stud. 2014;51(9): 1271-1280.

71. Chilcot J. The importance of illness perception in end-stage renal disease: associations with psychosocial and clinical outcomes. Semin Dial. 2012;25(1):59-64.

72. Leventhal H, Meyer D, Nerenz DR. The common sense representation of illness danger. In: Rachman S, editor. Contributions to Medical Psychology. New York: Pergamon Press; 1980:17-30. 
73. Clarke AL, Yates T, Smith AC, Chilcot J. Patient's perceptions of chronic kidney disease and their association with psychosocial and clinical outcomes: a narrative review. Clin Kidney J. 2016;9(3): 494-502.

74. Christensen AJ, Turner CW, Smith TW, Holman JM Jr, Gregory MC. Health locus of control and depression in end-stage renal disease. J Consult Clin Psychol. 1991;59(3):419-424.

75. Griva K, Jayasena D, Davenport A, Harrison M, Newman SP. Illness and treatment cognitions and health related quality of life in end stage renal disease. Br J Health Psychol. 2009;14(Pt 1): 17-34.

76. Knowles S, Swan L, Salzberg M, Castle D, Langham R. Exploring the relationships between health status, illness perceptions, coping strategies and psychological morbidity in a chronic kidney disease cohort. Am J Med Sci. 2014;348(4):271-276.

77. K/DOQI clinical practice guidelines for cardiovascular disease in dialysis patients. Am J Kidney Dis. 2005;45(4 Suppl 3):S1-S153.

78. Tong A, Sainsbury P, Carter SM, et al. Patients' priorities for health research: focus group study of patients with chronic kidney disease. Nephrol Dial Transplant. 2008;23(10):3206-3214.

79. Schipper K, Abma TA. Coping, family and mastery: top priorities for social science research by patients with chronic kidney disease. Nephrol Dial Transplant. 2011;26(10):3189-3195.

80. Lopes AA, Albert JM, Young EW, et al. Screening for depression in hemodialysis patients: associations with diagnosis, treatment, and outcomes in the DOPPS. Kidney Int. 2004;66(5):2047-2053.
81. Raymond CB, Wazny LD, Honcharik PL. Pharmacotherapeutic options for the treatment of depression in patients with chronic kidney disease. Nephrol Nurs J. 2008;35(3):257-263.

82. Palmer SC, Natale P, Ruospo M, et al. Antidepressants for treating depression in adults with end-stage kidney disease treated with dialysis. Cochrane Database Syst Rev. 2016;2016(5):CD004541.

83. Pascoe MC, Thompson DR, Castle DJ, McEvedy SM, Ski CF. Psychosocial interventions for depressive and anxiety symptoms in individuals with chronic kidney disease: systematic review and meta-analysis. Front Psychol. 2017;8:992.

84. Lerma A, Perez-Grovas H, Bermudez L, Peralta-Pedrero ML, Robles-García R, Lerma C. Brief cognitive behavioural intervention for depression and anxiety symptoms improves quality of life in chronic haemodialysis patients. Psychol Psychother. 2017;90(1):105-123.

85. Beck AT, Dozois DJ. Cognitive therapy: current status and future directions. Annu Rev Med. 2011;62:397-409.

86. Hudson JL, Moss-Morris R, Game D, Carroll A, Chilcot J. Improving distress in dialysis (iDiD): a tailored CBT self-management treatment for patients undergoing dialysis. J Ren Care. 2016;42(4):223-238.

87. NICE. Depression in adults with a chronic physical health problem. The NICE guideline on treatment and management. 2010. Available from: http://guidance.nice.org.uk/CG91/QuickRefGuide/pdf/English. Accessed October 9, 2017.

88. Finkelstein FO, Wuerth D, Finkelstein SH. An approach to addressing depression in patients with chronic kidney disease. Blood Purif. 2010;29(2):121-124.

\section{Publish your work in this journal}

The International Journal of Nephrology and Renovascular Disease is an international, peer-reviewed open access journal focusing on the pathophysiology of the kidney and vascular supply. Epidemiology, screening, diagnosis, and treatment interventions are covered as well as basic science, biochemical and immunological studies. The manuscript management system is completely online and includes a very quick and fair peer-review system, which is all easy to use. Visit http://www. dovepress.com/testimonials.php to read real quotes from published authors. 